\title{
Intestinal Response to Acute Intragastric and Intravenous Administration of Phosphate in Rats
}

\author{
Elena Layunta ${ }^{a, b}$ Eva Maria Pastor Arroyo ${ }^{a, b}$ Larissa Kägi ${ }^{a, b} \quad$ Linto Thomas ${ }^{a, b}$ \\ Moshe Levic Nati Hernando ${ }^{a, b}$ Carsten A. Wagner ${ }^{a, b}$ \\ alnstitute of Physiology, University of Zurich, Zurich, Switzerland, 'b Switzerland and National Center for \\ Competence in Research NCCR Kidney.CH, University of Zurich, Zurich, Switzerland, 'Department of \\ Biochemistry and Molecular \& Cellular Biology, Georgetown University, Washington, D.C., USA
}

\section{Key Words}

Intestine • Adaption • Phosphate • Transporter

\begin{abstract}
Background/Aims: Phosphate (Pi) homeostasis is controlled by the intestine and kidneys whose capacities to transport $\mathrm{Pi}$ are under endocrine control. Several studies point to intestinal absorption as a therapeutic target to modulate Pi homeostasis. The small intestine is responsible for almost all $\mathrm{Pi}$ absorption in the gut, a process involving $\mathrm{Na}^{+}$-dependent and independent mechanisms. Three $\mathrm{Na}^{+}$-dependent $\mathrm{Pi}$ cotransporters have been described in the gastrointestinal tract: NaPi-Ilb (a SLC34 member) and Pit-1 and Pit-2 (SLC20 transporters). We recently analysed the acute hormonal and renal response to intragastric (i.g) and intravenous (i.v) Pi-loading. This study demonstrated that the kidney quickly adapts to Pi-loading, with changes manifesting earlier in the i.v than i.g intervention. The aim of this work was to extend the previous studies in order to investigate the acute adaptation of intestinal transport of $\mathrm{Pi}$ and expression of intestinal $\mathrm{Na}^{+} / \mathrm{Pi}$-cotransporters in response to acute Pi-loading. Methods: Duodenal and jejunal mucosa was collected 40 minutes and/or 4 hours after administration (i.g and i.v) of either $\mathrm{NaCl}$ or $\mathrm{Pi}$ to anaesthetized rats. Uptakes of $\mathrm{Pi}$ and protein expression of $\mathrm{Na}^{+} / \mathrm{Pi}$ cotransporters were measured in brush border membrane vesicles (BBMV); the cotransporters' mRNA abundance was quantified by real-time PCR in total RNA extracted from whole mucosa. Results: Pi-loading did not modify transport of Pi in duodenal and jejunal BBMV 4 hours after treatment. Administration of Pi did not alter either the intestinal expression of NaPi-llb and Pit-2 mRNAs, whereas Pit-1 mRNA expression was only regulated (diminished) in duodenum collected 4 hours after i.g Pi-loading. NaPi-llb protein expression was decreased in duodenum 4 hours upon i.v Pi infusion, whereas the duodenal and jejunal abundance of
\end{abstract}


Layunta et al.: Acute Intestinal Response to Phosphate Load

the cotransporter was unaffected by i.g administration of Pi. Conclusion: Together, these data suggest that the intestine responds acutely to Pi-loading, though this response seems slower than the renal adaptation.

\section{Introduction}

Homeostasis of inorganic phosphate (Pi) is regulated by a wide range of hormonal mechanisms, which can trigger changes not only in renal Pi excretion but also in intestinal $\mathrm{Pi}$ absorption [1]. Intestinal absorption of dietary Pi takes place mainly in small intestine whereas the proximal tubule is the nephron segment mostly responsible for renal reabsorption [2].

Pi absorption in the small intestine depends on both a paracellular route driven by electrochemical gradients as well as on a $\mathrm{Na}^{+}$-dependent active transport. While the molecular mechanism(s) responsible for the paracellular route remain unknown, two families of $\mathrm{Na}^{+}-$ dependent Pi transporters are involved in the active intestinal Pi absorption, namely the SLC20 and the SLC34 families of solute carriers [3]; for review see [4, 5].

The SLC20 family consists of SLC20A1/Pit-1 and SLC20A2/Pit-2, two ubiquitously expressed proteins originally identified as viral receptors but later on shown to work as $\mathrm{Na}^{+} / \mathrm{Pi}$ cotransporters [6-8]. Cotransport mediated by Pit-1 and Pit-2 is electrogenic, as consequence of coupling transport of $2 \mathrm{Na}^{+}$ions with one monovalent $\mathrm{Pi}\left(\mathrm{H}_{2} \mathrm{PO}_{4}^{-}\right)$, with both transporters sharing similar apparent affinities for $\mathrm{Na}^{+}$and $\mathrm{Pi}$ (approximately $50 \mathrm{mM}$ and $100 \mu \mathrm{M}$, respectively) [9]. Pit-1 and Pit-2 transcripts are ubiquitously expressed [10]. In the small intestine of rats, both proteins are detected preferentially in duodenum and jejunum and their abundance is regulated by dietary Pi [11-13]. Although the contribution of SLC20 cotransporters to active intestinal Pi absorption has not been directly tested, indirect evidences suggest only a modest role $[14,15]$. A more recent study proposed the presence of additional systems involved in intestinal Pi-handling [13].

The SLC34 family consists of three members, with SLC34A1/NaPi-IIa and to a minor extent SLC34A3/NaPi-IIc mediating renal reabsorption, whereas SLC34A2/NaPi-IIb has an essential role in the active Pi uptake into enterocytes. NaPi-IIb is an electrogenic carrier which transports 3 molecules of $\mathrm{Na}^{+}$together with a molecule of $\mathrm{HPO}_{4}^{2-}$ with apparent affinities of about $25 \mathrm{mM}$ and $10 \mu \mathrm{M}$, respectively [16]. In addition to intestine, NaPi-IIb mRNA is expressed at high levels in many organs including lungs, salivary glands, uterus, mammary gland, testis, thyroid gland and placenta [10]. In mice, intestinal NaPi-IIb expression is mainly observed in ileum, being lower in jejunum and undetectable in duodenum [17]. However, in rats, this cotransporter is expressed mostly in jejunum, with lower levels in duodenum and absent from ileum [12]. In agreement with this pattern of NaPi-IIb expression, maximum Pi absorption in mice has been reported in ileum whereas the proximal segments exhibit higher transport activities in rats. Higher Pi absorption in duodenum than in ileum was also reported in humans $[18,19]$. Both full ablation [14] and intestinal specific depletion [15] of $\mathrm{NaPi}-\mathrm{IIb}$ in mice lead to a decrement of intestinal Pi absorption and increased faecal Pi loss. Moreover, NaPi-IIb may contribute to up to $50 \%$ of total Pi transport in the first hour after dietary intake [14], being the main Pi transporter in intestine described so far.

We and others have recently described that acute adaptation to intragastric (i.g) and intravenous (i.v) Pi-loading in rats associates with a fast phosphaturic response and that this response depends upon parathyroid hormone (PTH) release [20-22]. Moreover, we showed that the phosphaturia induced by Pi-overload correlates with reduced transport of $\mathrm{Pi}$ into renal brush border membrane vesicles (BBMVs) and reduced expression of NaPiIIc and/or NaPi-IIa, with all these effects being more pronounced in the i.v route but also observed in the i.g loading protocol. In contrast to intact animals, rats with surgical removal of parathyroid glands were unable to eliminate the Pi-load [21]. However, the effect of the previous manoeuvres on intestinal Pi transport and expression of intestinal cotransporters 
has not been studied. Therefore, the aim of the present work is to analyse the intestinal response to acute administration of $\mathrm{Pi}$.

\section{Materials and Methods}

\section{Animal Experimental Protocol}

The treatment of animals and all surgical procedures have been reported previously [21]. Briefly, male Wistar rats (Charles River, Wilmington, MA) with a weight between 250-350 g were adapted during 5 days to a diet containing low Pi ( $0.1 \%$, Kliba AG, Switzerland). To facilitate the transit of intragastrically administered solutions (see below), rats were fasted during the last 12 hours, with free access to water; to prevent coprophagy, fasting was performed in metabolic cages. Animals were anesthetized with $3 \%$ isoflurane/air, and kept on a warm pad with a temperature between $37-38^{\circ} \mathrm{C}$ while continuously inhaling a low dose of anesthesia (1-2\% isoflurane/air). Upon catheterization (BPE-T 50; Instech) of the femoral vein, a Ringer's solution $\left(116 \mathrm{mM} \mathrm{NaCl}, 1.2 \mathrm{mM} \mathrm{KCl}, 1 \mathrm{mM} \mathrm{CaCl}\right.$ and $2.7 \mathrm{mM} \mathrm{NaHCO}_{3}$ ) containing $5 \%$ glucose was infused continuously through the femoral vein at a rate of $3.0 \mathrm{ml} / \mathrm{hr}$ until the end of the experiment. Rats were randomly distributed into 4 experimental groups that were either intravenously infused with saline, intravenously infused with $\mathrm{Pi}$, intragastrically gavaged with saline or intragastrically gavaged with Pi. For the infusion protocol, one ml of either 150 or $500 \mathrm{mM} \mathrm{NaCl} \mathrm{pH} 7.4$ or $500 \mathrm{mM} \mathrm{Na} 2 \mathrm{HPO}_{4} / \mathrm{NaH}_{2} \mathrm{PO}_{4}$ pH 7.4 was administered intravenously. In the case of the gavage method, one $\mathrm{ml}$ of the above solutions adjusted at $\mathrm{pH} 5$ was dispensed directly into the stomach using a gavage tube (FTP-15-100, Instech). In some animals, the arrival of the bolus to the small intestine was visually confirmed by addition of a food colorant into the applied solution. For both protocols, applications were terminated 40 minutes or 4 hours postadministration; animals were sacrificed, duodenum and jejunum were dissected, rinsed vigorously with cold PBS, and inverted for the collection of the mucosa by scraping. The mucosal samples were immediately frozen in liquid nitrogen and stored at $-80^{\circ} \mathrm{C}$ until being used.

All procedures were carried out according the Swiss laws of animal protection and all protocols were previously approved by the local Veterinary Authority (Kantonales Veterinäramt Zürich).

\section{Isolation of intestinal brush border membrane vesicles (BBMVs) and flux measurements}

Flux measurements were done in freshly isolated BBMV. For that, samples of frozen duodenal and jejunal mucosa were homogenized in a buffer containing $300 \mathrm{mM}$ mannitol, $5 \mathrm{mM}$ EGTA and $12 \mathrm{mM}$ Tris$\mathrm{HCl}, \mathrm{pH} 7.1$, supplemented with protease and phosphatase inhibitors (Roche). BBMVs were then isolated by the $\mathrm{Mg}^{++}$-precipitation technique as reported previously [13], and finally resuspended in a buffer containing $300 \mathrm{mM}$ mannitol and $15 \mathrm{mM}$ HEPES-Tris, $\mathrm{pH}$ 7.4. Uptakes of ${ }^{32} \mathrm{P},{ }^{3} \mathrm{H}$-glucose and ${ }^{14} \mathrm{C}$-leucine (only in the jejunal experiments) into BBMVs were measured in two different solutions containing resuspension buffer supplemented with either $125 \mathrm{mM} \mathrm{NaCl}$ or $125 \mathrm{mM} \mathrm{KCl}$. In addition, these solutions contained either ${ }^{32} \mathrm{P}$, ${ }^{3} \mathrm{H}$-glucose or ${ }^{14} \mathrm{C}$-leucine as tracers, and $0.125 \mu \mathrm{M} \mathrm{K} \mathrm{HPO}_{4} / \mathrm{KH}_{2} \mathrm{PO}_{4} \mathrm{pH} 7.4,0.125 \mu \mathrm{M}$ D-glucose or $125 \mu \mathrm{M}$ leucine as cold substrate, respectively.

The incorporations of ${ }^{32} \mathrm{P}$, ${ }^{3} \mathrm{H}$-glucose and ${ }^{14} \mathrm{C}$-leucine were determined by incubating $10 \mu \mathrm{l}$ of freshly prepared BBMVs with $40 \mu \mathrm{l}$ of the different uptake solutions. Reactions were left to proceed for 1 minute and 2 hours at $25^{\circ} \mathrm{C}$. After that, uptakes were stopped by transferring $20 \mu \mathrm{l}$ of reaction samples to $1 \mathrm{ml}$ of ice cold stop solution ( $100 \mathrm{mM}$ mannitol, $5 \mathrm{mM}$ Tris- $\mathrm{HCl}, 150 \mathrm{mM} \mathrm{NaCl}, 5 \mathrm{mM}$ Pi and $5 \mathrm{mM}$ glucose). These suspensions were vortexed and spotted onto $0.45 \mu \mathrm{m}$ nitrocellulose filters (Sartorius Stedim Biotech) that were immediately vacuum-washed with $10 \mathrm{ml}$ of ice cold stop solution to remove non-incorporated tracer. Filters were finally transferred to plastic vials and upon addition of scintillation medium (Perkin Elmer) the radioactivity incorporated into the BBMVs was measured using a $\beta$-counter (Packard, TRI-CARB 2900TR). Each measurement was carried out in triplicates. The remaining BBMVs were stored at $-80^{\circ} \mathrm{C}$ for further analyses (protein determination and Western blots).

\section{RNA extraction and real-time quantitative RT-PCR}

Extraction of total RNA from frozen duodenal and jejunal mucosa was carried out with the RNeasy mini kit (Qiagen) following the manufacturers indications. Total RNA (100 ng) was then used as a template for first-strand cDNA synthesis by standard methods (TaqMan Reverse Transcription Kit from Applied 


\section{Cellular Physiology Cell Physiol Biochem 2019;52:838-849 \\ \begin{tabular}{ll|l} 
and Biochemistry & $\begin{array}{l}\text { DOl: 10.33594/000000058 } \\
\text { Published online: } 5 \text { April } 2019\end{array}$ & $\begin{array}{l}\text { O 2019 The Author(s). Published by } \\
\text { Cell Physiol Biochem Press GmbH\&Co. KG }\end{array}$ \\
\cline { 2 - 3 }
\end{tabular}}

Layunta et al.: Acute Intestinal Response to Phosphate Load

Biosystems). The resultant cDNA was subjected to PCR amplification of NaPi-IIb, Pit-1 and Pit-2 using the TaqMan Universal PCR Master Mix, in the presence of specific forward (fw) and reverse (rev) primers and FAM/TAMRA-labelled probes. The expression of the hypoxanthine phosphoribosyltransferase (HPRT) was used for calibration. The sequence of all primers and probes is shown below. The absence of genomic DNA contamination in the RNA preparation was controlled by PCR amplification of RNA samples without previous reverse transcription. Each reaction was run in duplicate and the mean Ct was calculated. Relative mRNA expression of each gene of interest under each experimental condition was expressed as $\Delta \mathrm{CT}=\mathrm{Ct}_{\mathrm{gene}^{-}}$ $\mathrm{Ct}_{\text {calibrator }}$

NaPi-IIb/fw: TCAGGTCCACAGTCTAGCATACCA

NaPi-IIb/rev: CGGAATATGAGGGTAGAAGTTCAATC

NaPi-IIb/probe: ACCAGCAAAACAAATGACAGCGGGAC

Pit-1/fw: CCTGCTTTGGGTCCTTTGC

Pit-1/rev: CAGCCACAGGGCCACAAG

Pit-1/probe: TGGCCTTCCCATCAGCACAACACATT

Pit-2/fw: CCTGCTTTGGGTCCTTTGC

Pit-2/rev: CAGCCACAGGGCCACAAG

Pit-2/probe: ACGGTGGCAACGATGTGAGCAATG

HPRT/fw: GCTGAAGATTTGGAAAAGGTGTTA

HPRT/rev: ACACAGAGGGCCACAATGTGA

HPRT/probe: TTATGGACAGGACTGAAAGACTTGCTCGAGATG

\section{Immunoblotting}

Protein levels of NaPi-IIb in BBMVs from duodenum and jejunum were quantified by immunoblotting. For that, the protein content in BBMVs was first determined by standard methods using commercially available reagents (BioRad protein determination kit). Samples containing $20 \mu \mathrm{g}$ of protein were denatured in Laemmli buffer, electrophoresed in 9\% SDS-PAGE gels and then transferred to polyvinylidene difluoride (PVDF) membranes (EMD Millipore) by electroblotting. PVDF membranes were blocked for 30 minutes with $5 \%$ non-fat dried milk in Tris-buffered saline containing $0.1 \%$ Tween-20, and sequentially incubated first with a rabbit polyclonal antibody against NaPi-IIb (1:1000; overnight at $\left.4{ }^{\circ} \mathrm{C}\right)$ [12] and then with a mouse monoclonal antibody against $\beta$-tubulin (1:25000; overnight at $4 \stackrel{\circ}{\circ}$ ). After washing and blocking again during 30 minutes, membranes were incubated during 2 hours at room temperature with the appropriated (anti-rabbit or anti-mouse) secondary antibody (Promega). Finally, membranes were overlaid with the chemiluminescent substrate for 5 minutes and protein was detected on a LAS-4000 luminescent image analyser (Fujifilm). Protein expression was calculated using Quantity One Analysis Software (BioRad) and the abundance of NaPi-IIb was normalized to the expression of $\beta$-Tubulin.

\section{Statistical analysis}

All results are expressed as mean \pm the standard error of the mean (SEM). Statistical comparisons were performed using either student's t-test and one-way ANOVA, followed by the Bonferroni post-test with a confidence interval of $95 \%(\mathrm{p}<0.05)$, as indicated. Statistical analysis was carried out with the computerassisted GraphPad Prism Program (Prism version 5.0, GraphPad Software, San Diego, CA).

\section{Results}

We recently showed that i.v administration of $\mathrm{Pi}$ to anesthetized rats blunted the incorporation of Pi into renal BBMVs, an effect that manifested 4 hours post administration, whereas no clear effect on Pi transport was detected 4 hours after i.g Pi-application [21]. 
Here, we extended these studies to analyse the intestinal response to Pi-loading in the same animals. For clarity, Table 1 contains an extract of the main physiological findings already reported in the previous manuscript [21].

\section{Effect of acute administration of Pi on ${ }^{32} \mathrm{P}$ uptake into duodenal and jejunal BBMVS}

To study the acute effect of Pi-loading in intestinal handling of $\mathrm{Pi}$, we first measured the transport of ${ }^{32} \mathrm{P}$ and ${ }^{3} \mathrm{H}$-glucose into BBMVs isolated from duodenal and jejunal mucosa collected 40 minutes and/or 4 hours after both Pi-administration protocols. Due to the large scattering of the uptake values, and since glucose incorporation has been shown to remain constant in some states with altered Pi-transport $[15,21]$, uptakes of ${ }^{32} \mathrm{P}$ were normalized to the incorporation of ${ }^{3} \mathrm{H}$-glucose. Although this normalization precluded the comparison of uptakes in duodenum with those of the jejunum (higher duodenal than jejunal uptakes have been previously reported [12]), it allowed us to compare the effect of Pi-loading in each intestinal segment.

As shown in Fig. 1A, the normalized transport into duodenal BBMVs was similar in samples from control $(\mathrm{NaCl})$ and Pi-loaded rats collected 4 hours after treatment. This lack of effect of Pi-loading on intestinal Pi-transport was observed both in the i.g and i.v protocols. Moreover, similar transport values were also observed in jejunal BBMVs of control and Piloaded rats collected 40 minutes as well as 4 hours post-administration (Fig. 1B).

Effect of acute administration of Pi on the duodenal and jejunal mRNA expression of $\mathrm{Na}^{+}$/ Pi-cotransporters

As indicated above, three $\mathrm{Na}^{+}$-dependent Pi cotransporters are expressed in small intestinal epithelial cells, namely NaPi-IIb, Pit-1 and Pit-2. It has been reported that in rats fed normal chow, the expression of NaPi-IIb at the mRNA and protein level is restricted to duodenum and jejunum (being higher in the later segment), whereas the cotransporters transcript and protein are undetectable in ileum [12]. In contrast, under the same dietary conditions the abundance of Pit-1 mRNA increases from the proximal to the distal part of the small intestine, though only the two most proximal segments express the protein [12]. Similar low expression of Pit-2 transcripts has been described in all small intestinal segments of rats fed normal chow [12].

Here, the mRNA expression of the three cotransporters was analysed by real time PCR in samples from duodenum and jejunum of rats subjected to i.v and i.g administration of $\mathrm{NaCl}$ or Pi. The duodenal and jejunal mRNA expression of NaPi-IIb was comparable in rats loaded with Pi or saline (Fig. 2A, B). Similar findings were observed in samples collected $40 \mathrm{~min}$ and 4 hours after phosphate loading. This analysis also indicated that there are no significant differences on NaPi-IIb mRNA abundance between duodenum and jejunum. The expression of Pit- 1 transcripts was significantly reduced in samples of duodenum collected 4 hours after i.g administration of Pi compared with the control group (Fig. 2C); no differences in Pit-1 mRNA abundance between $\mathrm{NaCl}$ and Pi-loaded rats were detected in the other conditions (Fig. 2C, D). Although the abundance of Pit-2 mRNA was slightly higher in duodenum than jejunum, the expression of this cotransporter was insensitive to Pi-loading (Fig. 2E, F).

\section{Effect of acute administration of Pi on the duodenal and jejunal protein abundance of} NaPi-IIb

We have recently reported that Pi-loading in rats modifies the protein abundance of $\mathrm{NaPi}-\mathrm{II} \mathrm{a}$ and NaPi-IIc, the two Slc34 $\mathrm{Na} / \mathrm{Pi}$ cotransporters expressed in renal BBM. NaPiIIa abundance was downregulated 4 hours after i.g and i.v Pi loading, whereas NaPi-IIc expression was reduced only in kidneys collected 4 hours after Pi i.v infusion [21].

Here, we have measured the abundance of NaPi-IIb at the protein level in BBM from duodenum and jejunum collected 4 hours upon loading (i.g and i.v) with $\mathrm{Pi}$ and $\mathrm{NaCl}$. The specificity of the antibody was confirmed by incubating BBM isolated from ileum of wild type and intestinal-specific NaPi-IIb deficient mice [15] (data not shown). In duodenal BBM of rats NaPi-IIb was detected as a smear concentrated at around $100 \mathrm{kDa}$ and its expression 


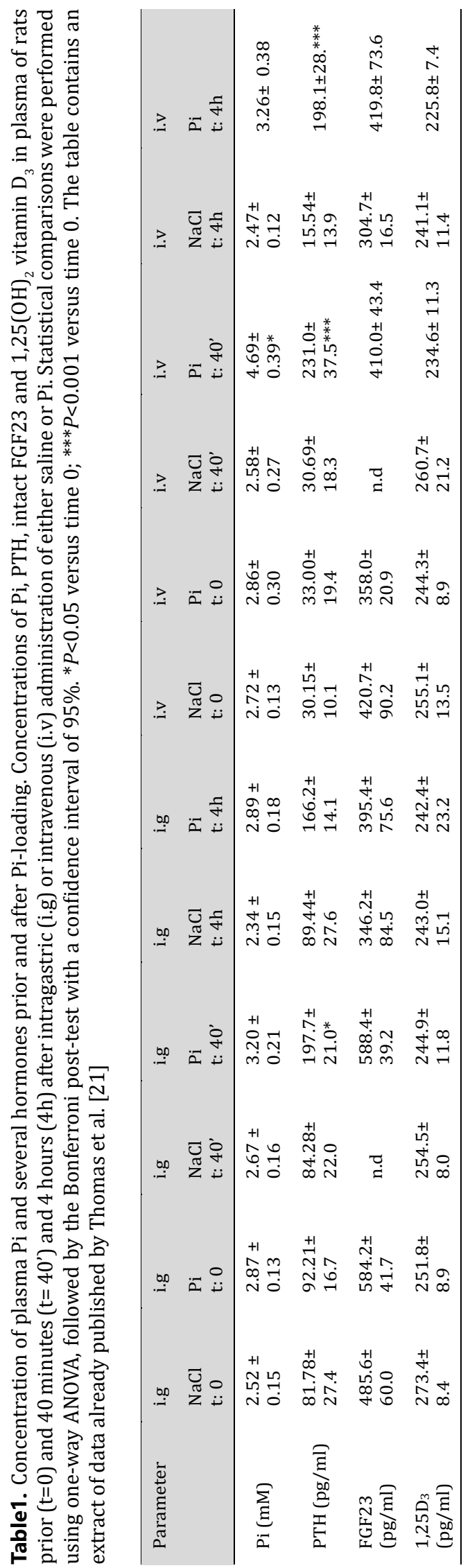

was not affected by i.g Pi-loading (Fig. 3A). This failure of the i.g administration of $\mathrm{Pi}$ to promote changes in the expression of $\mathrm{NaPi}-\mathrm{IIb}$ is in contrast with previous studies describing that in rats, an acute increment of $\mathrm{Pi}$ in the diet increases duodenal Pi transport as well as NaPi-IIb protein expression in the absence of mRNA changes [12]. As for the transport data discussed above, this discrepancy may be due to the slower intestinal transit of the i.g bolus in anesthetized compared with conscious animals. Nevertheless, since this manoeuvre did trigger a reduction on the renal expression of NaPi-IIa [21], the absence of NaPi-IIb regulation upon i.g Piloading suggests that the intestinal Slc34 cotransporter responds slower to changes in $\mathrm{Pi}$ homeostasis compared with the renal isoform. In contrast to the i.g administration, i.v infusion of $\mathrm{Pi}$ induced a significant reduction in the duodenal expression of NaPi-IIb (Fig. 3B). Reduced expression in the i.v Pi-loaded group was also observed upon normalization of the cotransporter abundance to the expression of $\mathrm{B}^{0} \mathrm{AT} 1$, a $\mathrm{Na}^{+}$-dependent neutral amino acid cotransporter expressed in renal and intestinal epithelia (data not shown). Together with the mRNA data (Fig. $2 \mathrm{~A}$ ), these results suggest post-transcriptional downregulation of duodenal NaPi-IIb in the i.v Pi-infused animals, although no differences in intestinal Pi absorption were yet noticed (Fig. 1A). In this regard, discrepancies between cotransporter abundance and Pi-uptakes into intestinal BBMV have been already detected: although the expression of NaPi-IIb seems by far higher in jejunum, the transport activity is larger in duodenum, a difference that may be due to segmental-specific posttranslational modifications and/or the contribution of additional transporters [12]. Moreover, discrepancies between cotransporter abundance (NaPi-IIa) and Pi-uptakes have been also reported in renal BBMV $[25,26]$.

In contrast to duodenal samples, $\mathrm{NaPi}-\mathrm{IIb}$ was detected as a broad smear (between 100 and $150 \mathrm{kDa}$ ) in BBM isolated from jejunal mucosa, and although a tendency for reduced protein expression was observed in animals subjected to i.g Pi-loading (Fig. 4A) this change was not significant. 

A) Duodenal phosphate and glucose transport

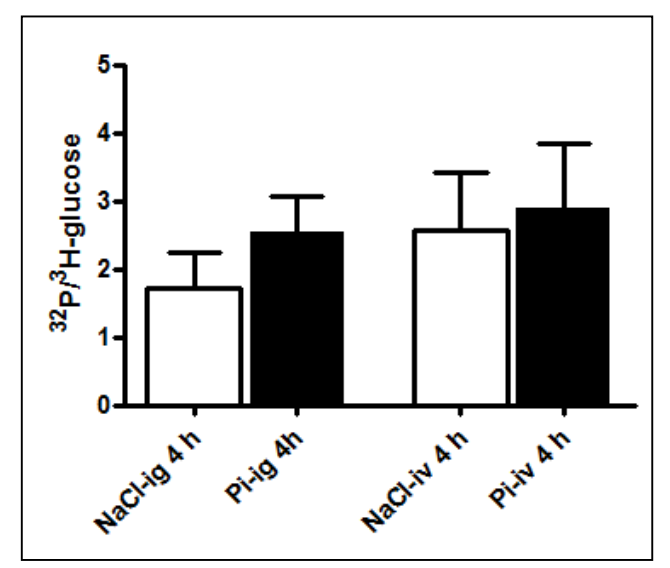

B) Jejunal phosphate and glucose transport

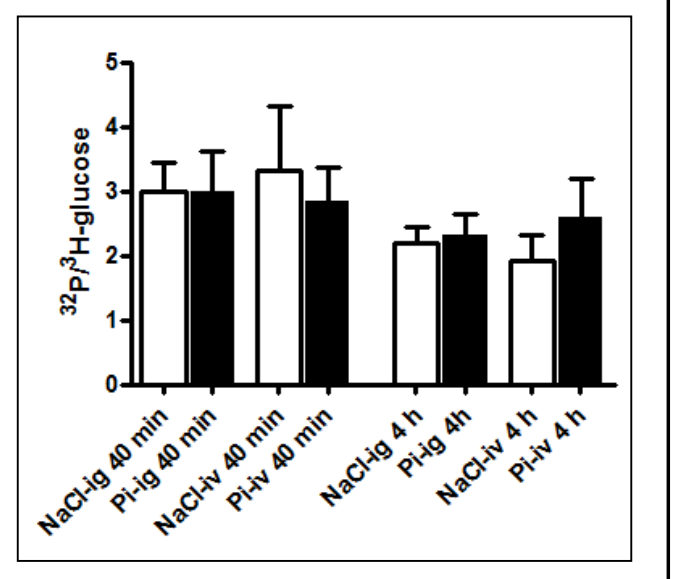

Fig. 1. Effect of acute administration of $\mathrm{Pi}$ on ${ }^{32} \mathrm{P}$ uptake into duodenal and jejunal BBMVs. Freshly prepared brush border membrane vesicles (BBMVs) from duodenal (A) and jejunal mucosa (B) of rats intragastrically gavaged with saline (NaCl-ig, $n \geq 5$ ) or with $\mathrm{Pi}(\mathrm{Pi}-\mathrm{ig}, \mathrm{n} \geq 10$ ) as well as of rats intravenously infused with saline (NaCl-iv, $n \geq 7$ ) or with $\mathrm{Pi}\left(\mathrm{Pi}-\mathrm{iv}, \mathrm{n} \geq 5\right.$ ) were incubated with ${ }^{32} \mathrm{P}$ and ${ }^{3} \mathrm{H}$-glucose in the presence of cold substrate as described in Material and Methods. For each rat, the $\mathrm{Na}^{+}$-dependent incorporation of ${ }^{32} \mathrm{P}$ was normalized to the $\mathrm{Na}^{+}$-dependent incorporation of ${ }^{3} \mathrm{H}$-glucose. Columns represent mean \pm the standard error of the mean (SEM). Statistical comparisons were performed using one-way ANOVA, followed by the Bonferroni post-test with a confidence interval of $95 \%$.

\section{Discussion}

Here we studied the rapid adaption of intestinal transcellular Pi transport in rats loaded with phosphate by gavage or i.v.. Regardless of the route, Pi-loading of anesthetized rats did not acutely regulate the Na+-dependent incorporation of Pi neither in duodenal nor in jejunal BBMVs. This is unlike the Pi transport into renal BBMVs whose activity was reduced 4 hours after i.v Piadministration [21], suggesting that the renal and intestinal response to changes on plasma levels of Pi proceed with different time courses. On the other hand, the absence of changes inintestinal transport observed in the i.g protocol is in contrast with previous reports showing that the acute change (4 hours) to high Pi-containing chow of rats previously fed 5 days with low Pi-containing diet triggers a substantial increase in the transport of Pi into jejunal and/or duodenal BBMVs $[12,13]$. This apparent discrepancy could be eventually due to the slower intestinal transit of the i.g bolus in anesthetized animals (this study) as compared with the normal transit of the chyme in the conscious state (previous studies) or may indicate that other cofactors present in normal animal chow present are required. Supporting a potential role of the slower intestinal transit in these differences, the acute feeding on high-Pi diet of rats previously adapted to low dietary Pi was shown to trigger a 3 fold increase in plasma Pi levels already 2 hours post-feeding, with hyperphosphatemia persisting after 4 hours [12] whereas we observed no differences in the circulating concentration of Pi upon intragastric administration of Pi to anaesthetized rats [21] (Table 1). Of note, we instilled a solution containing phosphate readily absorbable (and probably rapidly cleared by kidneys) whereas changes in dietary intake using rodent chow may also affect absorption rates and local phosphate concentrations.

While our study is the first one addressing the effect of fast changes in plasma Pi (induced by the i.v protocol) on the expression of intestinal transporters, the results of the i.g loading protocol are in agreement with previous studies showing that in rats, acute changes in dietary Pi do not modify NaPi-Ilb at the transcriptional level neither in duodenum nor in 
A) NaPi-Ilb mRNA in Duodenum

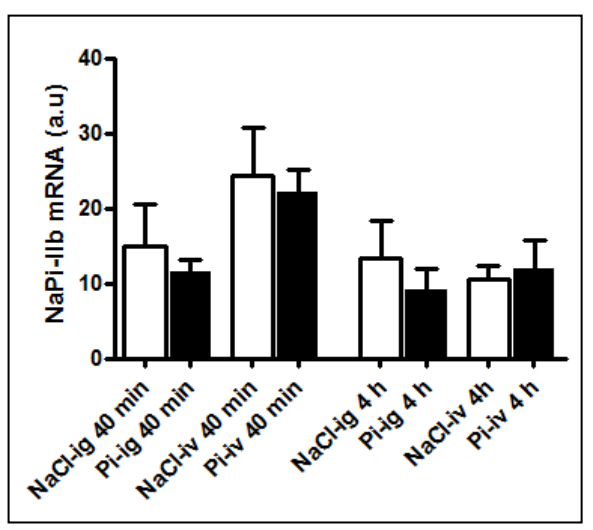

C) Pit1 mRNA in Duodenum

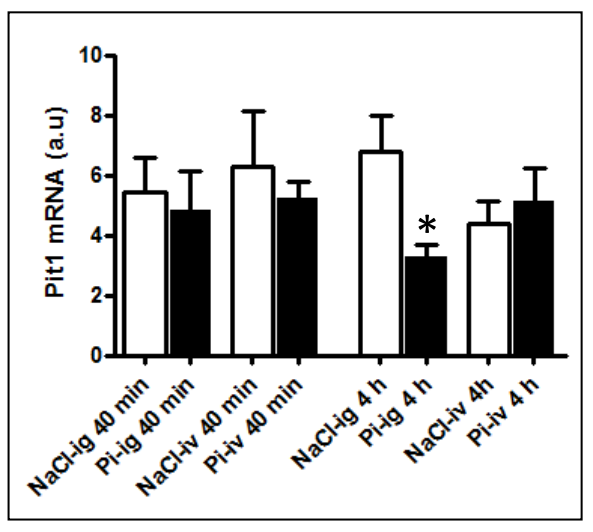

E) Pit2 mRNA in Duodenum

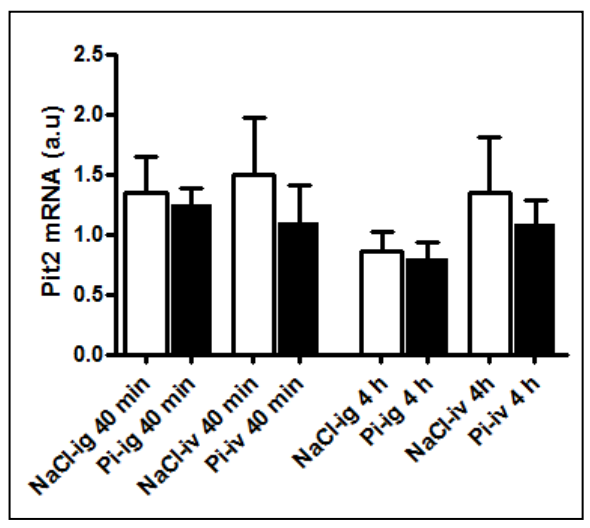

B) NaPi-Ilb mRNA in Jejunum

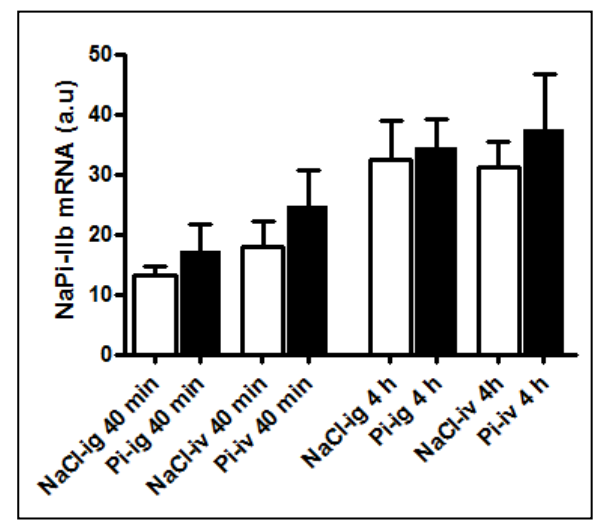

D) Pit1 mRNA in Jejunum

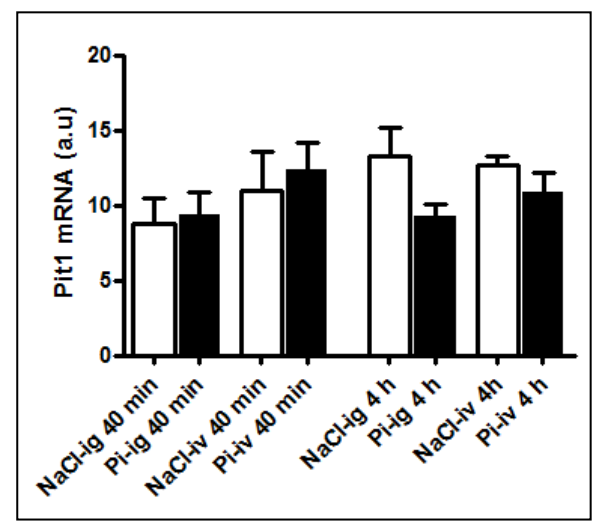

F) Pit2 mRNA in Jejunum

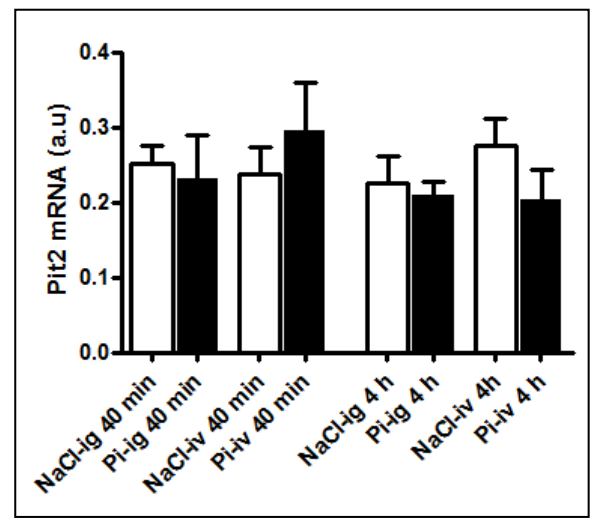

Fig. 2. Effect of acute administration of $\mathrm{Pi}$ on the duodenal and jejunal mRNA expression of $\mathrm{Na}^{+} / \mathrm{Pi}^{-}$ cotransporters. The mRNA expression of NaPi-IIb (A, B), Pit-1 (C, D) and Pit-2 (E, F) was quantified by realtime RT-PCR in samples from duodenum $(A, C, E)$ and jejunum $(B, D, F)$ of rats intragastrically gavaged with saline (NaCl-ig) or with Pi (Pi-ig) as well as of rats intravenously infused with saline (NaCl-iv) or with Pi (Piiv). Samples were collected either 40 minutes ( $40 \mathrm{~min}$ ) or 4 hours ( $4 \mathrm{~h}$ ) post-administration. The expression of each cotransporter was normalized to the abundance of HPRT. Columns represent mean \pm the standard error of the mean (SEM); $n=5$ for the 40 minutes and $n \geq 8$ for the 4 hours groups. Statistical comparisons were performed using one-way ANOVA, followed by the Bonferroni post-test with a confidence interval of $95 \%$. ${ }^{*}<0.05$ compared with the corresponding saline value. 


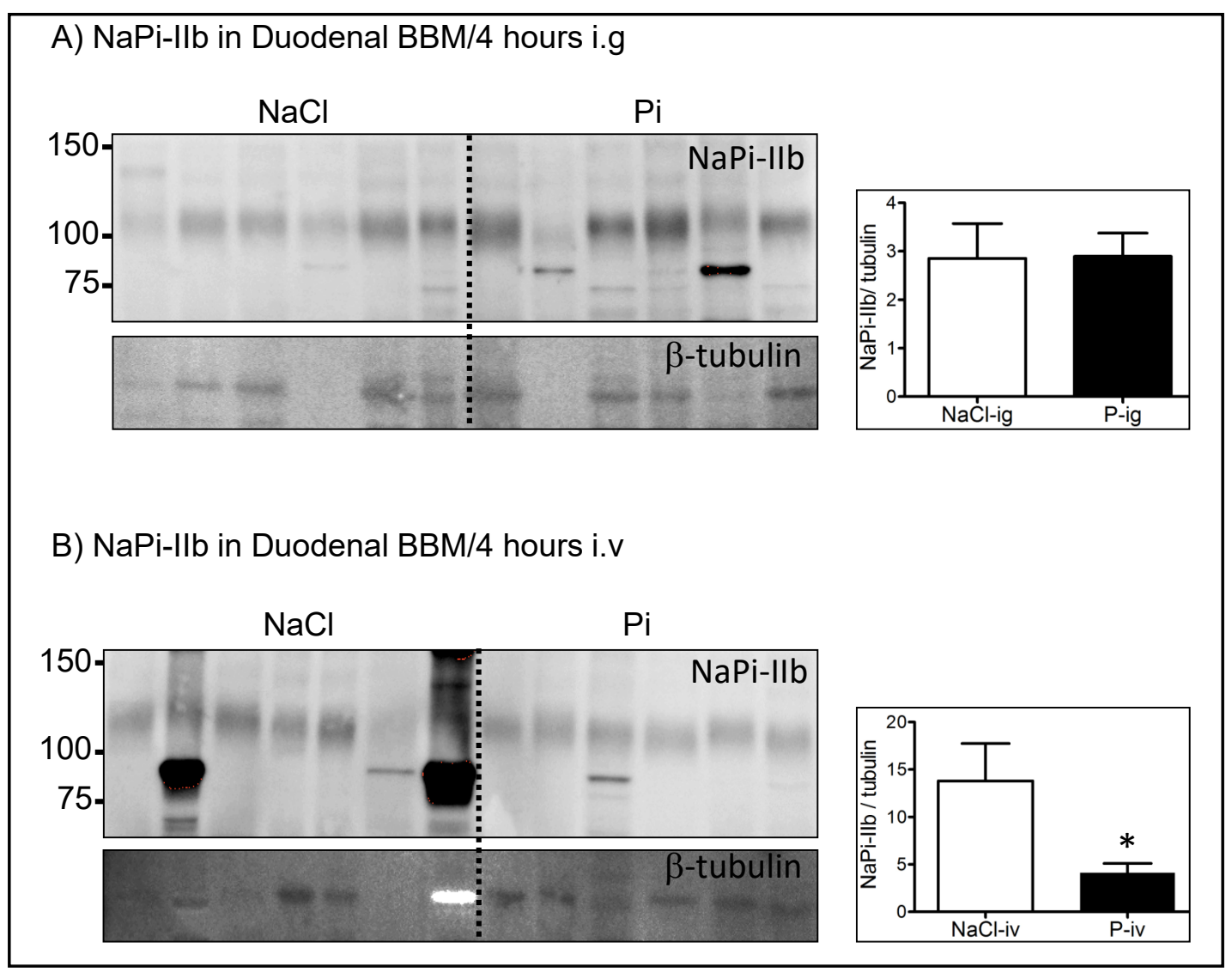

Fig. 3. Effect of acute administration of $\mathrm{Pi}$ on the duodenal NaPi-IIb protein abundance. The protein expression of NaPi-IIb was analysed in (BBMVs) from duodenal mucosa of rats intragastrically (A) or intravenously (B) infused with saline $(\mathrm{NaCl})$ or with $\mathrm{Pi}(\mathrm{n} \geq 6)$. $\beta$-tubulin was used as control for proteinloading. The figure shows representative blots and normalized NaPi-IIb/ $\beta$-tubulin expression. Columns in the graphs represent mean \pm the standard error of the mean (SEM). Statistical comparisons were performed using student's t-test. ${ }^{*} \mathrm{p}<0.05$ compared with the corresponding control value.

jejunum [12]. In contrast to acute changes, chronic feeding of low Pi is known to associate with higher expression of NaPi-IIb mRNA both in rats [12] and mice [17, 23]. In rats this upregulation is found specifically in jejunum, whereas the transcript expression in duodenum remains unaffected [12]. As mentioned above, the intestinal abundance of NaPi-IIb mRNA has been reported to be higher in jejunum than duodenum of rats fed standard food [12]. In our study rats were fed with low Pi diet for 5 days prior to the administration of the $\mathrm{NaCl} / \mathrm{Pi}$ load, which may explain the absence of consistent differences on NaPi-IIb mRNA expression between both intestinal segments. On the other hand, our findings regarding the expression of Pit- 1 and Pit- 2 are in agreement with several publications reporting that neither acute [12] nor chronic changes $[12,24]$ of dietary Pi alter the mRNA expression of intestinal Slc20 cotransporters in rats.

This failure of the i.g administration of Pi to promote changes in the expression of $\mathrm{NaPi}-\mathrm{IIb}$ is in contrast with previous studies describing that in rats, an acute increment of $\mathrm{Pi}$ in the diet increases duodenal Pi transport as well as NaPi-IIb protein expression in the absence of mRNA changes [12]. As for the transport data discussed above, this discrepancy may be due to the slower intestinal transit of the i.g bolus in anesthetized compared with conscious animals. Nevertheless, since this manoeuvre did trigger a reduction on the renal expression of NaPi-IIa [21], the absence of NaPi-IIb regulation upon i.g Pi-loading suggests that the intestinal Slc34 cotransporter responds slower to changes in Pi homeostasis compared with the renal isoform. 
A) NaPi-Ilb in Jejunal BBM/4 hours i.g
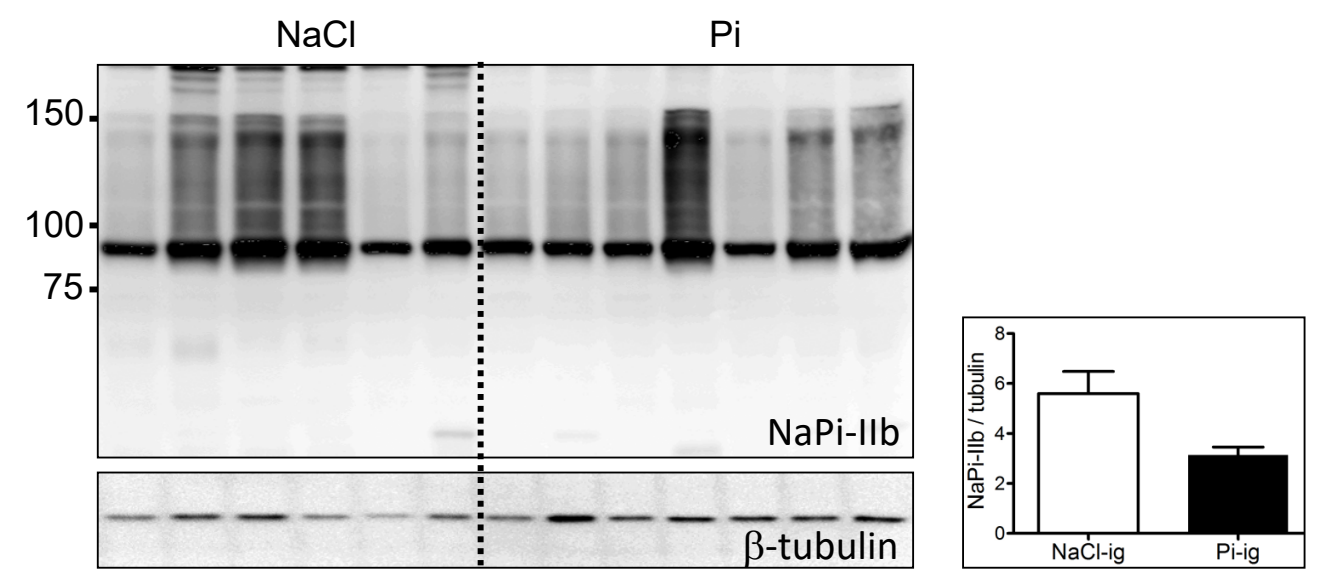

B) NaPi-Ilb in Jejunal BBM/4 hours i.v
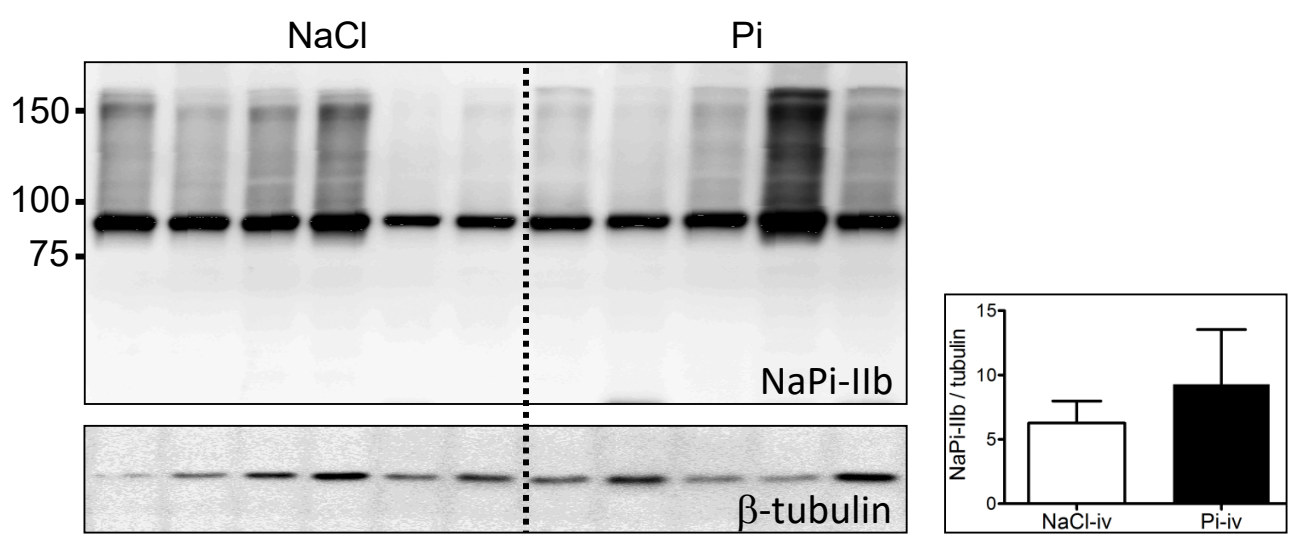

Fig. 4. Effect of acute administration of Pi on the jejunal NaPi-IIb protein abundance. The expression of $\mathrm{NaPi}$-IIb protein was analysed in (BBMVs) from jejunal mucosa of rats intragastrically (A) or intravenously (B) infused with saline $(\mathrm{NaCl})$ or with $\mathrm{Pi}(\mathrm{n} \geq 5)$. $\beta$-tubulin was used as control for protein-loading. The figure shows representative blots and normalized NaPi-IIb/ $\beta$-tubulin expression. Columns in the graphs represent mean \pm the standard error of the mean (SEM). Statistical comparisons were performed using student's t-test. ${ }^{*} \mathrm{p}<0.05$ compared with the corresponding control value.

We found similar NaPi-IIb levels in the jejunum in control and Pi-loaded rats with both protocols. Our data is in agreement with previous data showing no differences in jejunal $\mathrm{NaPi}-\mathrm{IIb}$ protein expression in rats upon acute dietary changes [12]. Thus, while jejunal (but not duodenal) expression of $\mathrm{NaPi}$-IIb is specifically regulated in response to chronic changes on dietary Pi in rats [12], the duodenum rather than the jejunum seems to respond to acute changes ([12] and this work). Moreover, the duodenal response was detected upon i.v but not i.g Pi administration. Among other parameters, a main difference between both protocols was that plasma Pi did not change upon i.g Pi-loading whereas the expected transient increase was detected upon i.v Pi-infusion (Table 1 and [21]). These findings, together with the short transit time in duodenum suggest that the triggering signal is not luminal Pi but rather transient hyperphosphatemia. The different responsiveness of duodenal and jejunal $\mathrm{NaPi}$-IIb to both acute and chronic changes on dietary/plasma Pi may suggests that both intestinal segments are equipped with different sensor/effector molecules. 
Layunta et al.: Acute Intestinal Response to Phosphate Load

\section{Conclusion}

In summary, we have shown that i.v Pi-load but noti.g Pi-administration to anaesthetized rats acutely downregulates the expression of NaPi-IIb in duodenal BBM. These results suggest that intestinal adaptation to changes in Pi homeostasis may take longer than the renal adaptation, since both loading manoeuvres were reported to alter the expression of NaPi-IIa in renal proximal tubules [21]. Transient hyperphosphatemia may be responsible for the acute downregulation of duodenal NaPi-IIb abundance of i.v Pi-loaded rats, though further experiments are required to properly address this issue.

\section{Acknowledgements}

The authors acknowledge the support of the Zurich Integrative Rodent Physiology Core Facility. This work was supported by a grant from the National Center for Competence in Research (NCCR) Kidney.CH, Switzerland, to J.Biber and C.A Wagner. Aragon Regional Government and IBERCAJA-CAI supported the predoctoral research stay of E. Layunta in the Institute of Physiology at the University of Zurich.

\section{Disclosure Statement}

The authors declare that they have no competing financial interests.

\section{References}

1 Lederer E: Regulation of serum phosphate. J Physiol 2014;592:3985-3995.

2 Sabbagh Y, Giral H, Caldas Y, Levi M, Schiavi SC: Intestinal phosphate transport. Adv Chronic Kidney Dis 2011;18:85-90.

3 Forster IC, Hernando N, Biber J, Murer H: Phosphate transporters of the SLC20 and SLC34 families. Mol Aspects Med 2013;34:386-395.

4 Biber J, Hernando N, Forster I: Phosphate transporters and their function. Annu Rev Physiol 2013;75:535550 .

5 Forster I, Hernando N, Sorribas V, Werner A: Phosphate transporters in renal, gastrointestinal, and other tissues. Adv Chronic Kidney Dis 2011;18:63-76.

6 Kavanaugh MP, Miller DG, Zhang W, Law W, Kozak SL, Kabat D, Miller AD: Cell-surface receptors for gibbon ape leukemia virus and amphotropic murine retrovirus are inducible sodium-dependent phosphate symporters. Proc Natl Acad Sci U S A 1994;91:7071-7075.

7 Collins JF, Bai L, Ghishan FK: The SLC20 family of proteins: dual functions as sodium-phosphate cotransporters and viral receptors. Pflugers Arch 2004;447:647-652.

8 Olah Z, Lehel C, Anderson WB, Eiden MV, Wilson CA: The cellular receptor for gibbon ape leukemia virus is a novel high affinity sodium-dependent phosphate transporter. J Biol Chem 1994;269:25426-25431.

9 Ravera S, Virkki LV, Murer H, Forster IC: Deciphering PiT transport kinetics and substrate specificity using electrophysiology and flux measurements. Am J Physiol Cell Physiol 2007;293:C606-620.

10 Nishimura M, Naito S: Tissue-specific mRNA expression profiles of human solute carrier transporter superfamilies. Drug Metab Pharmacokinet 2008;23:22-44.

11 Bai L, Collins JF, Ghishan FK: Cloning and characterization of a type III Na-dependent phosphate cotransporter from mouse intestine. Am J Physiol Cell Physiol 2000;279:C1135-1143.

12 Giral H, Caldas Y, Sutherland E, Wilson P, Breusegem S, Barry N, Blaine J, Jiang T, Wang XX, Levi M: Regulation of rat intestinal Na-dependent phosphate transporters by dietary phosphate. Am J Physiol Renal Physiol 2009;297:F1466-F1475.

13 Candeal E, Caldas YA, Guillen N, Levi M, Sorribas V: Intestinal phosphate absorption is mediated by multiple transport systems in rats. Am J Physiol Gastrointest Liver Physiol 2017;312:G355-G366. 


\section{Cellular Physiology Cell Physiol Biochem 2019;52:838-849 \begin{tabular}{ll|l} 
and $10.33594 / 000000058$ & O 2019 The Author(s). Published by \\
Cell Physiol Biochem Press GmbH\&Co. KG
\end{tabular}}

Layunta et al.: Acute Intestinal Response to Phosphate Load

- 14 Sabbagh Y, O’Brien SP, Song W, Boulanger JH, Stockmann A, Arbeeny C, Schiavi SC: Intestinal npt2b plays a major role in phosphate absorption and homeostasis. J Am Soc Nephrol 2009;20:2348-2358.

- 15 Hernando N, Myakala K, Simona F, Knopfel T, Thomas L, Murer H, Wagner CA, Biber J: Intestinal Depletion of NaPi-IIb/Slc34a2 in Mice: Renal and Hormonal Adaptation. J Bone Miner Res 2015; DOI:10.1002/ jbmr.2523.

- 16 Forster IC, Virkki L, Bossi E, Murer H, Biber J: Electrogenic kinetics of a mammalian intestinal type IIb $\mathrm{Na}(+) / \mathrm{P}(\mathrm{i})$ cotransporter. J Membr Biol 2006;212:177-190.

- 17 Radanovic T, Wagner CA, Murer H, Biber J: Regulation of intestinal phosphate transport. I. Segmental expression and adaptation to low-P(i) diet of the type IIb $\mathrm{Na}(+)-\mathrm{P}(\mathrm{i})$ cotransporter in mouse small intestine. Am J Physiol Gastrointest Liver Physiol 2005;288:G496-500.

18 Juan D, Liptak P, Gray TK: Absorption of inorganic phosphate in the human jejunum and its inhibition by salmon calcitonin. J Clin Endocrinol Metab 1976;43:517-522.

19 Walton J, Gray TK: Absorption of inorganic phosphate in the human small intestine. Clin Sci (Lond) 1979;56:407-412.

20 Scanni R, vonRotz M, Jehle S, Hulter HN, Krapf R: The human response to acute enteral and parenteral phosphate loads. J Am Soc Nephrol 2014;25:2730-2739.

21 Thomas L, Bettoni C, Knopfel T, Hernando N, Biber J, Wagner CA: Acute Adaption to Oral or Intravenous Phosphate Requires Parathyroid Hormone. J Am Soc Nephrol 2017;28:903-914.

- 22 Lee GJ, Mossa-Al Hashimi L, Debnam ES, Unwin RJ, Marks J: Postprandial adjustments in renal phosphate excretion do not involve a gut-derived phosphaturic factor. Exp Physiol 2017;102:462-474.

- 23 Capuano P, Radanovic T, Wagner CA, Bacic D, Kato S, Uchiyama Y, St-Arnoud R, Murer H, Biber J: Intestinal and renal adaptation to a low-Pi diet of type II NaPi cotransporters in vitamin D receptor- and 1alphaOHase-deficient mice. Am J Physiol Cell Physiol 2005;288:C429-434.

24 Katai K, Miyamoto K, Kishida S, Segawa H, Nii T, Tanaka H, Tani Y, Arai H, Tatsumi S, Morita K, Taketani Y, Takeda E: Regulation of intestinal Na+-dependent phosphate co-transporters by a low-phosphate diet and 1, 25-dihydroxyvitamin D3. Biochem J 1999;343:705-712.

25 Nowik M, Picard N, Stange G, Capuano P, Tenenhouse HS, Biber J, Murer H, Wagner CA: Renal phosphaturia during metabolic acidosis revisited: molecular mechanisms for decreased renal phosphate reabsorption. Pflugers Arch 2008;457:539-549.

26 Zajicek HK, Wang H, Puttaparthi K, Halaihel N, Markovich D, Shayman J, Beliveau R, Wilson P, Rogers T, Levi M: Glycosphingolipids modulate renal phosphate transport in potassium deficiency. Kidney Int 2001;60:694-704. 